\title{
Ensino da avaliação psicológica no Norte brasileiro: analisando as ementas das disciplinas
}

\author{
Leogildo Alves Freires ${ }^{1}$ \\ Universidade Federal de Roraima, Boa Vista-RR, Brasil \\ José Humberto da Silva Filho \\ Universidade Federal do Amazonas, Manaus-AM, Brasil \\ Renan Pereira Monteiro \\ Universidade Federal do Piauí, Parnaíba-PI, Brasil \\ Gleidson Diego Lopes Loureto, Valdiney Veloso Gouveia \\ Universidade Federal da Paraíba, João Pessoa-PB, Brasil
}

\section{RESUMO}

O presente estudo objetivou analisar as ementas das disciplinas de avaliação psicológica (AP) dos cursos de graduação do Norte brasileiro. Inicialmente, observou-se uma pluralidade de denominações no campo da AP nessa Região, e que os cursos de graduação ofertam, em média, 3,82 disciplinas nessa área, sendo que o contato inicial se dá a partir do segundo ano do curso. Com relação ao conteúdo das ementas, apenas seis instituições o disponibilizaram, sendo tais informações analisadas com o software Iramuteq. Os resultados indicaram um ementário que destaca a formação de caráter tecnicista com ênfase na centralidade do psicodiagnóstico, nos procedimentos de aplicação e interpretação de instrumentos e em construtos tradicionais, como inteligência e personalidade. Pode-se concluir que existe a necessidade de empreender esforços para alinhar o ensino da AP no Norte às diretrizes dessa área no Brasil e aos avanços teóricos, técnicos e metodológicos da atualidade.

Palavras-chave: ensino da psicologia; avaliação psicológica; formação profissional.

\section{ABSTRACT - Teaching of psychological assessment in Northern of Brazil: Analyzing the courses' summaries}

This study aimed at analyzing the syllabuses of psychological assessment (PA) disciplines offered in higher education Psychology courses in the North of Brazil. Initially, it was possible to verify a variety of denominations for the field of PA in this region and that these courses offer an average of 3.82 disciplines in this area. The initial contact of the students with PA occurs in the sophomore year. Only six institutions provided the content of the syllabuses, and this data was analyzed with a software named Iramuteq. The results indicated a syllabus that features a technicist training trend, emphasizing the psychological diagnostic practice, the procedures of application and interpretation of instruments and traditional constructs such as intelligence and personality. In conclusion, the need for efforts to align the teaching of PA in the North with the guidelines of this field in Brazil and to the current theoretical, technical and methodological advances is clear.

Keywords: psychology teaching; psychological assessment; professional education.

RESUMEN - Enseñanza de la evaluación psicológica en el Norte brasileño: análisis de las descripciones de las asignaturas Este estudio ha tenido como objetivo analizar las descripciones de las asignaturas de evaluación psicológica (EP) de las licenciaturas del norte brasileño. Inicialmente, se ha observado que existe una pluralidad designativa en el ámbito de la EP en esta zona geográfica y que las referidas licenciaturas ofrecen cerca de 3,82 asignaturas en este campo, siendo que el contacto inicial ocurre a partir del segundo año del curso. Con relación al contenido de las descripciones de las asignaturas, solamente seis instituciones se las han cedido. Para análisis de estas informaciones, se ha utilizado el software Iramuteq, cuyos resultados han indicado que el sumario de las asignaturas privilegia una formación de carácter tecnicista con énfasis en la centralidad del psicodiagnóstico, en los procedimientos de aplicación e interpretación de instrumentos y en los constructos tradicionales, como por ejemplo la inteligencia y personalidad. Se concluye que hay una necesidad de emprender esfuerzos para equiparar la enseñanza psicológica en el Norte con las directrices del área en Brasil y a los avances teóricos, técnicos y metodológicos actuales.

Palabras clave: enseñanza de psicología; evaluación psicológica; formación profesional. 
A avaliação psicológica (AP) é uma área fundamental para a Psicologia como ciência e profissão. Na prática profissional, a AP é uma ferramenta privativa do psicólogo, norteando sua atuação em contextos diversos, auxiliando-o na tomada de decisões e no planejamento de intervenções adequadas às demandas do solicitante (Noronha, Baldo, Barbin, \& Freitas, 2003; Nunes et al., 2012). Por outro lado, para o avanço do conhecimento científico em Psicologia, a AP, em sua vertente mais voltada para a elaboração de medidas, é um elemento indispensável, possibilitando a operacionalização de domínios psicológicos e, consequentemente, a testagem de hipóteses e teorias (Primi, 2010).

É evidente, pois, a importância do ensino da AP durante a formação na área da Psicologia. A propósito, esse campo do conhecimento psicológico é um dos eixos estruturantes para a formação de psicólogos, de acordo com as Diretrizes Curriculares Nacionais (Bardagi, Teixeira, Segabinazi, Schelini, \& Nascimento, 2015). Nessa direção, espera-se que ao longo da formação o discente desenvolva habilidades e competências básicas referentes à $\mathrm{AP}$, a exemplo da elaboração de documentos oriundos da avaliação, da construção e adaptação de instrumentos de medida e, ademais, de possuir noções básicas de estatística, fundamentando uma futura atuação profissional que o capacite também nessa área essencial (Noronha, Carvalho, Miguel, Souza, \& Santos, 2010).

Não obstante, observa-se por vezes, na prática, que a formação em AP apresenta problemas, sobretudo pela falta de sistematização dos principais aspectos a serem abordados durante o curso, resultando em uma formação inadequada na área (Noronha et al., 2012; Noronha \& Reppold, 2010). Buscando a sistematização do ensino da AP, Nunes et al. (2012) elaboraram um documento propondo diretrizes, destacando resoluções do Conselho Federal de Psicologia (CFP) e do Código de Ética Profissional do Psicólogo. O documento foi estruturado em quatro partes:

1. competências básicas a serem desenvolvidas ao longo da formação (e.g., ter conhecimento sobre funções, origem, natureza e uso dos testes em AP; ter conhecimento sobre validade, precisão, normatização e padronização de instrumentos psicológicos);

2. disciplinas e conteúdos programáticos respectivos, propondo cinco disciplinas e um estágio supervisionado em AP;

3. estrutura de ensino, contando com infraestrutura adequada, métodos de ensino para desenvolver habilidades nos estudantes, docentes com formação acadêmica, experiência e atualização na área; e, finalmente,

4. referências básicas indicadas para tais disciplinas.

A proposição de diretrizes para o ensino da $\mathrm{AP}$ justifica-se em razão de achados observados na literatura sobre a formação deficitária e o conhecimento incipiente de estudantes e profissionais que atuam nessa área. Concretamente, análises de provas do Exame Nacional de Desempenho dos Estudantes (ENADE) têm atestado o rendimento insatisfatório de estudantes de Psicologia especialmente em questões relacionadas com a área de medidas (Primi, Hutz, \& Silva, 2011; Souza, Bastos, \& Barbosa, 2011).

Outras evidências são disponibilizadas por Noronha, Nunes e Ambiel (2007), que verificaram que os estudantes dão pouca importância a questões fundamentais dessa área, como o conhecimento de ampla variedade de testes, a construção de instrumentos e noções de estatística. Esses autores também verificaram o domínio dos discentes em diferentes aspectos relativos à AP, observando médias inferiores em assuntos como psicopatologia, elaboração de pareceres, noções sobre construção de instrumentos e conhecimento de construtos. Especificamente no que se refere a noções de estatística, um dado que chamou a atenção foi a não variação do nível de domínio dos estudantes em função dos anos de graduação, o que é preocupante em razão de tais noções estarem estreitamente relacionadas com o entendimento de princípios elementares de psicometria.

Em outro estudo, dessa vez avaliando a percepção de docentes sobre a importância e o domínio de aspectos relacionados com a AP, Noronha, Barros, Nunes e Santos (2014) verificaram que os conteúdos tidos como menos importantes por esses profissionais - e sobre os quais não tinham domínio adequado - foram aqueles relacionados a noções básicas de estatística, embora também não tivessem demonstrado conhecimento de uma variedade de testes e conceitos clássicos em psicometria. Portanto, no conjunto, esses achados indicam certa similaridade entre discentes e docentes no que se refere aos aspectos valorizados e ao domínio de conteúdos considerados essenciais no âmbito da AP. Claro, não se pode descartar que parte importante do desempenho deficitário de estudantes em quesitos fundamentais da AP pode ser resultado da má formação recebida de seus professores.

De fato, alguns estudos vêm indicando um panorama nebuloso no que tange à qualidade da formação nessa área. Em pesquisa que buscou conhecer a opinião de estudantes de Psicologia sobre o ensino da AP, Paula, Pereira, e Nascimento (2007) observaram críticas referentes à insuficiência de conteúdo e ao reduzido número de disciplinas ofertadas. Em pesquisas com psicólogos também foi possível observar queixas no que tange à formação em AP recebida durante a graduação. Por exemplo, Padilha, Noronha, e Fagan (2007), contando com a participação de 85 psicólogos, verificaram que $60 \%$ indicaram não fazer uso de testes psicológicos, sendo que $43 \%$ apontaram a falta de conhecimento como um dos motivos para a não aplicação. Ainda, esses autores verificaram que cerca de $50 \%$ dos psicólogos julgaram insatisfatória a formação que tiveram em AP.

Outros resultados preocupantes nesse âmbito foram descritos por Mendes, Nakano, Silva, e Sampaio (2013). Essas autoras observaram que profissionais e estudantes não se diferenciam no que tange ao domínio 
de questões centrais da AP (e.g., conceituar AP, definir validade e precisão). Essa situação poderia não ser preocupante se a AP não fosse uma prática privativa do profissional de Psicologia, conferindo aos psicólogos elementos para definir sua identidade e especificidade laboral, e se os discentes não estivessem em processo de formação, reforçando problemas no ensino das habilidades correspondentes que implicam em seu desempenho deficitário (Mendes et al., 2013).

Quanto aos problemas enfrentados durante a formação, alguns docentes citam, por exemplo, a falta de local adequado para atividades práticas, o número reduzido de testes psicológicos disponíveis, a desatualização e as más condições de armazenamento e uso dos instrumentos (Bardagi et al., 2015). Dessa forma, há necessidade de refletir sobre o ensino de AP nos cursos de graduação em Psicologia, entendendo que as formações básica e continuada são fundamentais para atenuar preconceitos, em geral de ordens ética e técnica, que ainda permeiam a prática da AP no Brasil (Borsa, 2016; Gouveia et al., 2002). Além disso, em termos práticos - e para além dos aspectos supracitados —, é importante destacar o número reduzido de disciplinas voltadas para a AP em alguns cursos de graduação no país (Reppold \& Serafinni, 2010), sendo relevante ter em conta a análise da estrutura curricular dos cursos, checando disciplinas dedicadas à AP e seus conteúdos.

As informações apresentadas até o momento indicam que é importante discutir a formação em AP, procurando oferecer possibilidades de aprimorá-la. Diversos estudos nessa área têm se debruçado em análises sobre o ensino da avaliação no eixo Sul-Sudeste, havendo escassa evidência na literatura sobre o processo de formação em AP na região Norte, objeto de análise deste estudo. Um dos poucos estudos que consideraram essa região foi levado a cabo por Noronha (2006), que avaliou 41 ementas de disciplinas de AP de 38 cursos de graduação no Brasil, sendo a região Norte representada por apenas $2 \%$ do montante analisado. De modo geral, essa autora verificou a diversidade de nomenclatura das disciplinas em AP, sendo que em $78 \%$ dos cursos o primeiro contato dos estudantes com tais disciplinas ocorreu até o quarto período; a carga horária média das disciplinas foi de 226,61 horas, contando com aproximadamente 3,29 disciplinas dessa área durante a formação. Não foram oferecidas informações específicas para cada região.

Em outro estudo, Noronha et al. (2008) consideraram 39 ementas de disciplinas relacionadas com a AP em 14 universidades brasileiras, uma delas do Norte (Tocantins). Em consonância com o que foi observado antes, os autores comprovaram a pluralidade de nomenclatura das disciplinas. Em termos específicos, eles constataram que os conteúdos mais frequentes foram aqueles relacionados a técnicas projetivas, testes de personalidade, testes psicológicos e testes de inteligência. Por outro lado, os conteúdos relativos a instrumentos psicométricos, testes situacionais e avaliação psicomotora tiveram menor frequência.
Apesar da contribuição relevante desses estudos, pouco se sabe, concretamente, sobre a estruturação do ensino de AP na região Norte do Brasil. Esse tem sido um contexto pouco explorado e, por vezes, esquecido, sendo responsável por pouco menos de $10 \%$ dos cursos de graduação em Psicologia no país (Lisboa \& Barbosa, 2009). Observações sobre o panorama do ensino de AP no Norte evidenciam certo atraso em comparação com as demais regiões do país, sobretudo Sul, Sudeste e Nordeste. De acordo com informações do Instituto Brasileiro de Avaliação Psicológica (IBAP), existem apenas dois laboratórios de AP na Região Norte, ambos localizados no Amazonas [Laboratório de Avaliação Psicológica do Amazonas (Universidade Federal do Amazonas) e Laboratório de Medidas e Avaliação Psicológica (Faculdade Martha Falcão)]. Assim, a produção de conhecimento nessa área tem se concentrado, sobretudo, em um único estado, o que indica um cenário desfavorável ao desenvolvimento da AP na região. Em recente estudo que mapeou a produção científica nessa área entre 2003 e 2012, Santos, Anache, e Santana (2015) verificaram que a região Norte representou apenas $0,72 \%$ da produção nacional. Dessa forma, parece justificável e relevante ter em conta a formação em AP nessa região do país.

Por fim, em linha com os temas do VII Congresso Brasileiro de Avaliação Psicológica - que tratou dos desafios da AP nas diferentes regiões do Brasil - e do VIII Congresso Brasileiro de Avaliação Psicológica - que discutiu, no período de 2 a 5 de maio de 2017, a formação em AP —, parece fundamental conhecer como se configura o ensino nessa área na região Norte do país. Assim, o presente estudo objetivou conhecer a estruturação do ensino da AP nas instituições de ensino superior (IESs) da região Norte, analisando as ementas de suas disciplinas e as matrizes curriculares disponíveis.

\section{Método}

\section{Material}

Inicialmente, por meio de consulta ao site do Ministério da Educação (http://emec.mec.gov.br/), identificaram-se 36 cursos de graduação em Psicologia, sendo sete de instituições públicas e 29 de instituições particulares. Entretanto, neste estudo, consideraram-se os dados de apenas 28 cursos (seis de instituições públicas e 22 de instituições particulares). O critério utilizado foi a disponibilidade de acesso à matriz curricular e/ou ao ementário das disciplinas no endereço eletrônico das respectivas instituições. No que tange às matrizes curriculares, realizaram-se análises descritivas das disciplinas de AP. Em relação às ementas, desenvolveram-se análises mais robustas. Para esta análise, foram considerados seis cursos de Psicologia que disponibilizaram o ementário das disciplinas na íntegra. 


\section{Análise dos dados}

Os bancos textuais foram analisados por meio do software Iramuteq (Interface de $R$ pour les Analyses Multidimensionnelles de Textes et de Questionnaires; Ratinaud, 2009 ), que é ancorado no software $R$ ( $R$ Development Core Team, 2012) e na linguagem de programação python (www.python.org). Após a criação do corpus textual, composto apenas pelas ementas das disciplinas de AP por instituição de ensino, cada uma representando uma unidade de contexto inicial (UCI), foi realizada uma análise de classificação hierárquica descendente (CHD), na qual consideraram-se ativas as seguintes classes gramaticais: adjetivo, substantivo e verbo. Esse procedimento classifica os segmentos de texto em consonância com seus respectivos vocabulários, com base na frequência e no $\chi^{2}$, permitindo a criação de classes de unidades de contexto elementares (UCEs) formadas por classes lexicais que são caracterizadas por vocabulário e segmentos de textos específicos de um dado corpo textual (Camargo, 2005). Depois de criadas, as classes de UCEs foram nomeadas e descritas. Ademais, foram realizadas as análises de "nuvem de palavras" (agrupando e organizando-as graficamente em função de sua frequência) e a análise de similitude (a partir da identificação das coocorrências entre as palavras), que fornece indicações da conexidade entre as palavras, permitindo o reconhecimento da estrutura de um corpus (Ratinaud \& Marchand, 2012).

\section{Resultados}

Inicialmente, efetuou-se um levantamento acerca das disciplinas naárea de AP disponibilizadas pelos cursos de Psicologia (Tabela 1). Foi possível verificar a pluralidade de nomenclatura no âmbito da AP, contabilizando-se 56 disciplinas, sendo mais

Tabela 1

Frequência das disciplinas relativas à avaliação psicológica das instituições de ensino superior da região Norte do Brasil

\begin{tabular}{|c|c|c|c|}
\hline Disciplina & $\mathrm{F}$ & Disciplina & $\mathrm{F}$ \\
\hline Psicodiagnóstico & 19 & $\begin{array}{l}\text { Fundamentos da Avaliação Psicológica - Testes } \\
\text { Psicométricos e Projetivos }\end{array}$ & 1 \\
\hline Técnicas de Avaliação Psicológica I & 5 & Técnicas Projetivas I & 1 \\
\hline Técnicas de Avaliação Psicológica II & 5 & Introdução à Psicometria & 1 \\
\hline Técnicas de Exame Psicológico I & 4 & Técnicas Psicométricas & 1 \\
\hline Técnicas de Exame Psicológico II & 4 & Avaliação Psicológica III & 1 \\
\hline Psicometria & 3 & Testes Projetivos Gráficos e não Gráficos & 1 \\
\hline Avaliação Psicológica & 3 & Tópicos Especiais em Testes & 1 \\
\hline Avaliação Neuropsicológica & 3 & Práticas e Avaliação Psicológica - Testes Psicológicos & 1 \\
\hline Métodos e Técnicas de Avaliação Psicológica & 3 & Tópicos em Psicodiagnóstico & 1 \\
\hline Fundamentos das Medidas Psicológicas & 3 & Técnicas de Avaliação da Inteligência & 1 \\
\hline Técnicas Projetivas & 2 & Métodos e Técnicas de Avaliação Psicológica I & 1 \\
\hline Psicodiagnóstico Infantil & 2 & Métodos e Técnicas de Avaliação Psicológica II & 1 \\
\hline Avaliação Psicológica I & 2 & Técnicas de Avaliação Psicológica I & 1 \\
\hline Avaliação Psicológica II & 2 & Técnicas de Avaliação Psicológica II & 1 \\
\hline Testes Psicológicos I & 2 & Técnicas de Avaliação Psicológica III & 1 \\
\hline Testes Psicológicos II & 2 & Seminário Temático em Avaliação Psicológica I & 1 \\
\hline Instrumento e Avaliação em Psicologia I & 1 & Seminário Temático em Avaliação Psicológica II & 1 \\
\hline Medidas em Psicologia I & 1 & Seminário Temático em Avaliação Psicológica III & 1 \\
\hline Medidas em Psicologia II & 1 & Medidas de Avaliação em Psicologia I & 1 \\
\hline Instrumentos e Medidas em Psicologia I & 1 & Medidas de Avaliação em Psicologia II & 1 \\
\hline Instrumentos e Medidas em Psicologia II & 1 & Medidas de Avaliação em Psicologia III & 1 \\
\hline Instrumentos e Medidas em Psicologia III & 1 & Medidas de Avaliação em Psicologia IV & 1 \\
\hline Estágio Básico III - Psicodiagnóstico & 1 & Estágio em Avaliação em Psicologia I & 1 \\
\hline Práticas e Avaliação Psicológica - Estágio Básico VI & 1 & Estágio em Avaliação em Psicologia II & 1 \\
\hline Práticas e Avaliação Psicológica - Estágio Básico V & 1 & Estágio em Avaliação em Psicologia III & 1 \\
\hline Técnicas Projetivas Gráficas Infantis & 1 & Estágio em Avaliação em Psicologia IV & 1 \\
\hline
\end{tabular}

Nota: $\mathrm{F}=$ frequência. 
frequentes aquelas nomeadas como Psicodiagnóstico (19\%), Técnicas de Avaliação Psicológica I (5\%) e II (5\%) e Técnicas de Exame Psicológico I (4\%) e II (4\%).

No que se refere à carga horária dos cursos, observou-se média de 4.224 horas $(D P=315,42$; amplitude de 3,920 a 4,953 horas), sendo disponibilizadas, em média, 240 horas $(D P=11,20$; amplitude de 80 a 660 horas) para disciplinas voltadas à AP. A média de disciplinas nessa área oferecida pelos cursos foi de 3,82, sendo que uma das instituições do conjunto total analisado não apresentou disciplina relacionada com a AP. Verificou-se que um curso ofertou 11 disciplinas nesse campo do conhecimento psicológico. Outro achado importante foi que o primeiro contato dos estudantes com disciplinas dessa área, em quatro cursos especificamente, se deu a partir do segundo ano de graduação. Destaca-se que no presente estudo não foi feita qualquer distinção quanto à natureza das disciplinas (obrigatórias ou optativas).
Posteriormente, efetuou-se análise de CHD, por meio da qual se verificou que 22 dos cursos analisados informaram apenas o nome das disciplinas. Sendo assim, optou-se por levar em conta apenas o ementário de seis cursos que disponibilizaram as informações das ementas na íntegra. A CHD do corpus 1 foi formada por seis UCIs; o corpus foi dividido em 42 UCEs, das quais $79,2 \%$ foram consideradas na CHD, tendo sido analisadas 1.803 palavras. O corpus se dividiu em 7 classes de segmentos de textos, e foram descritas apenas as palavras cujos $\chi^{2}$ foram iguais ou superiores ao valor mínimo adequado $\left(\chi^{2} \geq 3,84\right)$, conforme pode ser observado no dendograma (Figura 1), em que são indicados os valores do $\chi^{2}$ entre parênteses, seguidos da frequência média de cada palavra.

A classe 1 foi denominada "construção de medidas" (12\% das UCEs), tendo em conta aspectos relacionados com a aplicação da estatística para a construção de medidas em Psicologia (e.g., validade, precisão, análise de

\begin{tabular}{|c|c|c|c|c|c|c|}
\hline & & & & & & \\
\hline \multirow{20}{*}{$\begin{array}{l}\text { Classe } 1 \\
5 \text { UCEs } \\
12 \% \\
\text { Construção } \\
\text { de medidas } \\
\text { Escala } \\
(28,3) 7 \\
\text { Medida } \\
(13,6) 15 \\
\text { Diferente } \\
(9,24) 4 \\
\text { Geral } \\
(9,24) 3 \\
\text { Psicologia } \\
(9,22) 19 \\
\text { Precisão } \\
(6,12) 7 \\
\text { Validade } \\
(4,27) 8\end{array}$} & \multirow{17}{*}{$\begin{array}{l}\quad \text { Classe } 2 \\
\quad 7 \text { UCEs } \\
16,7 \% \\
\\
\text { Aplicação e } \\
\text { interpretação } \\
\text { Clínica } \\
(16,3) 6 \\
\text { Interpretar } \\
(16,3) 5 \\
\text { Instrumento } \\
(10,5) 20 \\
\text { Técnica } \\
(10,3) 26 \\
\text { Aplicar } \\
(8,89) 13 \\
\text { Projetivo } \\
(7,67) 10 \\
\text { Área } \\
(5,82) 6 \\
\text { Psicométricos } \\
(5,82) 4 \\
\text { Psicometria } \\
(5,82) 4 \\
\text { Organizacional } \\
(5,82) 4 \\
\text { Escolar } \\
(5,82) 4\end{array}$} & \multirow{16}{*}{$\begin{array}{l}\quad \text { Classe } 5 \\
\quad 6 \text { UCEs } \\
14,3 \% \\
\text { Construtos } \\
\text { Personalidade } \\
(26,5) 6 \\
\text { Específico } \\
(13,3) 4 \\
\text { Cognitivo } \\
(9,69) 6 \\
\text { Aspecto } \\
(7,29) 10 \\
\text { Elaboração } \\
(5,93) 12 \\
\text { Projeto } \\
(4,61) 4 \\
\text { Execução } \\
(4,61) 4\end{array}$} & \multirow{16}{*}{\begin{tabular}{l}
\multicolumn{1}{c|}{ Classe 3} \\
$\quad 8$ UCEs \\
$14,3 \%$ \\
Entrevista \\
Entrevista \\
$(14,9) 8$ \\
Testes \\
psicológicos \\
$(12,1) 9$ \\
Psicológicos \\
$(10,2) 8$ \\
Aplicação \\
$(6,74) 18$ \\
Psicológica \\
$(4,75) 5$ \\
Interpretação \\
$(4,75) 4$ \\
Devolução \\
$(4,75) 4$ \\
Função \\
$(4,75) 3$
\end{tabular}} & \multirow{19}{*}{\begin{tabular}{l}
\multicolumn{1}{c}{ Classe 4} \\
\multicolumn{1}{c}{19 UCEs } \\
\\
Psicodiagnóstico \\
Campo \\
$(20,0) 6$ \\
Psicodiagnóstico \\
(18,7) 17 \\
Diagnóstico \\
(9,69) 9 \\
Utilizar \\
(7,24) 5 \\
Princípio \\
$(7,24) 4$ \\
Conceituação \\
(7,24) 4 \\
Outro \\
(7,24) 3 \\
Implicação \\
(7,24) 3 \\
Laudo \\
(7,09) 11 \\
Relatório \\
(7,09) 10 \\
Processo \\
$(6,07) 24$ \\
Temático \\
$(4,61) 4$ \\
Seminário \\
$(4,61) 4$
\end{tabular}} & \multirow{20}{*}{$\begin{array}{l}\text { Classe } 6 \\
\quad 5 \text { UCEs } \\
11,9 \% \\
\text { Planejamento } \\
\text { da sessão } \\
\text { Maneira } \\
(42,0) 7 \\
\text { Diverso } \\
\text { (34,0) } 8 \\
\text { Criterioso } \\
(34,0) 8 \\
\text { Bateria } \\
(34,0) 8 \\
\text { Psicoterápicos } \\
(32,7) 5 \\
\text { Avaliação } \\
\text { clínica } \\
(32,7) 5 \\
\text { Tipo } \\
(28,3) 13 \\
\text { Crítica } \\
(24,1) 10 \\
\text { Avaliação } \\
\text { psicológica } \\
(14,1) 33 \\
\text { Conhecer } \\
(14,9) 18 \\
\text { Teste } \\
(11,3) 20 \\
\text { Psicanálise } \\
(9,24) 3 \\
\text { Saúde } \\
(4,27) 5\end{array}$} & \multirow{17}{*}{ 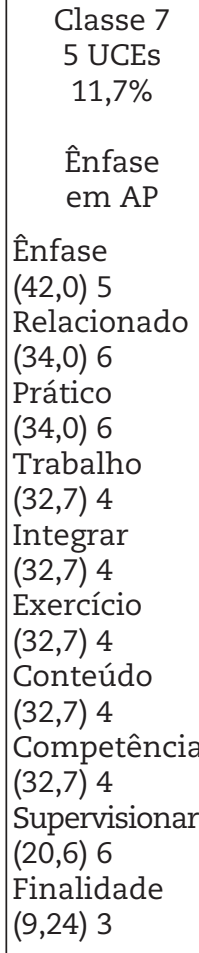 } \\
\hline & & & & & & \\
\hline & & & & & & \\
\hline & & & & & & \\
\hline & & & & & & \\
\hline & & & & & & \\
\hline & & & & & & \\
\hline & & & & & & \\
\hline & & & & & & \\
\hline & & & & & & \\
\hline & & & & & & \\
\hline & & & & & & \\
\hline & & & & & & \\
\hline & & & & & & \\
\hline & & & & & & \\
\hline & & & & & & \\
\hline & & & & & & \\
\hline & & & & & & \\
\hline & & & & & & \\
\hline & & & & & & \\
\hline
\end{tabular}

Figura 1. Dendograma da classificação hierárquica descendente do corpus 1 
itens), ressaltando, também, as potencialidades e limitações de medição nessa área. Ademais, a classe 1 abordou aspectos conceituais no âmbito da psicometria, a exemplo da teoria da medida. Essa classe pode ser ilustrada a partir dos seguintes trechos: "conhecer as teorias gerais de medidas. Compreender os processos relacionados à precisão, validade e análise de itens. Conhecer, identificar, caracterizar e construir escalas de medidas e seu uso nos diferentes tipos de investigação em Psicologia"; e "estatística aplicada à Psicologia. Elaborar e validar escalas de medidas psicológicas e/ou instrumentos de avaliação".

A classe 2 foi definida como "aplicação e interpretação" (17,6\% das UCEs), referindo-se ao emprego da instrumentação psicológica (técnicas projetivas e objetivas) nos mais variados contextos, tais como clínico, organizacional e escolar. Os seguintes trechos ilustram essa classe: "a utilização de instrumentos psicométricos, testes psicológicos, inventários e escalas para a avaliação de construtos psicológicos em diferentes contextos de atuação organizacional, desenvolvimento, clínica, dentre outros"; e "conceituar e conhecer os aspectos históricos, os objetivos, a validade e a precisão das técnicas expressivas gráficas. Ser capaz de identificar, aplicar e interpretar os tipos de técnicas projetivas gráficas".

A classe 3, nomeada como "entrevista" $(14,3 \%$ das UCEs), retrata essa técnica como parte do processo de AP, relacionando-a com outras formas de coleta de informação, enfatizando, ainda, o momento em que é empregada, seja no início do processo de AP ou na devolutiva. Os fragmentos a seguir ilustram tal classe: "técnicas de entrevista focadas na análise da cognição. Testes psicométricos: princípios éticos, aplicação, análise dos resultados e entrevista de devolução, considerando as análises quantitativa e qualitativa dos resultados"; e "modelos de entrevista na avaliação psicológica. Técnicas de observação do comportamento. Planejamento da avaliação psicológica. Organização da bateria de testes psicológicos. Integração de informações na avaliação psicológica”.

A classe 4 foi rotulada de "psicodiagnóstico" (19,1\% das UCEs), percebendo-se uma perspectiva deste enquanto processo sistematizado, incluindo diferentes etapas de execução. Observa-se, também, a menção aos documentos oriundos do processo de AP, tais como relatório, parecer e laudo psicológico. Aspectos éticos envolvidos nesse processo foram mencionados. Os trechos a seguir exemplificam a presente classe: "a dialética do normal e do patológico. Diagnóstico clínico. Princípios de análise e interpretação. Documentos emitidos pelo psicólogo, parecer, relatório e laudo psicológico. Comunicação dos resultados. Implicações práticas e éticas do psicodiagnóstico"; e "elaboração de laudos dos testes, aptidões específicas e de interesses. Implicações éticas e requisitos legais".

A classe 5 foi denominada "construtos" $(14,3 \%$ das UCEs), abrangendo fenômenos psicológicos que são alvo de avaliação, como aspectos cognitivos (inteligência), personalidade, ansiedade, depressão, atenção concentrada e aspectos psicomotores. Apresentam-se dois fragmentos do texto que ilustram tal classe: "inteligência; habilidades cognitivas; personalidade; funções neuropsicológicas; saúde geral; ansiedade e depressão; e atenção concentrada; além de testes destinados à seleção de motoristas"; e "testes para a avaliação das funções cognitivas: Gestáltico Visomotor de Bender, WISC-III (Escala de Inteligência Wechsler para Crianças), Matrizes Progressivas de Raven, DFH (Desenho da Figura Humana: avaliação do desenvolvimento cognitivo de crianças brasileiras - DFH III)".

A classe 6 recebeu a etiqueta "planejamento da sessão" (11,9\% das UCEs), enfatizando a estruturação da AP. Destacam-se, ainda, aspectos voltados ao conhecimento e ao domínio do instrumental psicológico e seu uso a partir de uma visão crítica, conforme indicam os fragmentos a seguir: "conhecer os diversos tipos de testes e baterias para avaliação psicológica, aplicando-os de maneira criteriosa e crítica"; e "Técnicas de Avaliação Psicológica I. História da psicometria: principais questões envolvidas na testagem psicológica no Brasil e no mundo. Classificação e administração de instrumentos psicométricos. Perspectiva crítica e ética para o uso de testes psicológicos".

Por fim, a classe 7 recebeu a denominação "ênfase em AP" (11,7\% das UCEs), denotando a priorização dessa área de atuação, cobrindo as ementas de uma IES específica que oferece ênfase em AP, formada por sete disciplinas, três delas do núcleo comum, quatro da ênfase em AP e quatro de estágios na área. Portanto, alguns dos fragmentos versam sobre atividades práticas e de intervenção em AP, como explicitam os seguintes trechos: "trabalhos práticos supervisionados voltados para o desenvolvimento integrado e o exercício das competências e habilidades relacionadas aos conteúdos da ênfase"; e "elaboração de um projeto de intervenção, execução e relatório".

Conhecidas as classes que surgiram a partir da análise das ementas, optou-se por verificar as palavras mais frequentemente utilizadas no corpus, recorrendo-se, para tanto, à "nuvem de palavras" (Figura 2). Os termos que ocuparam lugar de maior destaque, sendo os mais frequentes, foram: avaliação psicológica, técnica, instrumento, processo, psicodiagnóstico, teste, aplicação, conhecer e medida.

Por fim, efetuou-se análise de similitude (Figura 3), seguindo o mesmo padrão de intuito da análise anterior, que possibilita a visualização de coocorrências das palavras e, ainda, as indicações de conexão entre elas. Observa-se que a árvore de coocorrência é composta de alguns núcleos centrais, a partir dos quais emergem as ramificações. Concretamente, além da centralidade do termo avaliação psicológica, palavras como avaliação, técnica, processo e psicodiagnóstico também obtiveram destaque na análise, atuando na organização dos demais elementos do corpus, isto é, as ementas das disciplinas de AP das IESs. Ou seja, essas são as palavras mais presentes no banco de dados textuais (ementário das disciplinas) 
e, ainda, as responsáveis pelas conexões com as demais palavras do corpus analisado.

\section{Discussão}

O objetivo do presente estudo foi conhecer a estruturação do ensino de AP nos cursos de Psicologia da região Norte do Brasil, analisando ementas e matrizes curriculares. O que se verificou, inicialmente, foi a diversidade de nomenclatura das disciplinas, fenômeno já observado em estudos prévios e que pode indicar divergências entre os cursos, refletindo a possível falta de sistematização acerca dos aspectos centrais a serem abordados durante a formação (Noronha, 2006, Noronha, Carvalho, Miguel, Souza, \& Santos, 2010; Noronha et al., 2007, 2008).
No que se refere à estrutura do ensino, os cursos disponibilizaram, em média, carga horária superior a 200 horas para disciplinas de AP, sendo identificada média de mais de três disciplinas voltadas para esse campo de atuação, corroborando os resultados descritos em outros estudos (Castro, 2013; Noronha, 2006). Esses resultados evidenciam o distanciamento das diretrizes elaboradas por Nunes et al. (2012). Segundo os autores, os cursos de Psicologia devem ofertar em sua estrutura curricular pelo menos cinco disciplinas e a possibilidade de estágio supervisionado. Entretanto, cabe destacar que apenas uma dentre as instituições analisadas enfatizou a AP em seu currículo, contando com sete disciplinas e quatro estágios na área. Não obstante, tomando os dados em sua totalidade, observa-se que a realidade encontrada nos

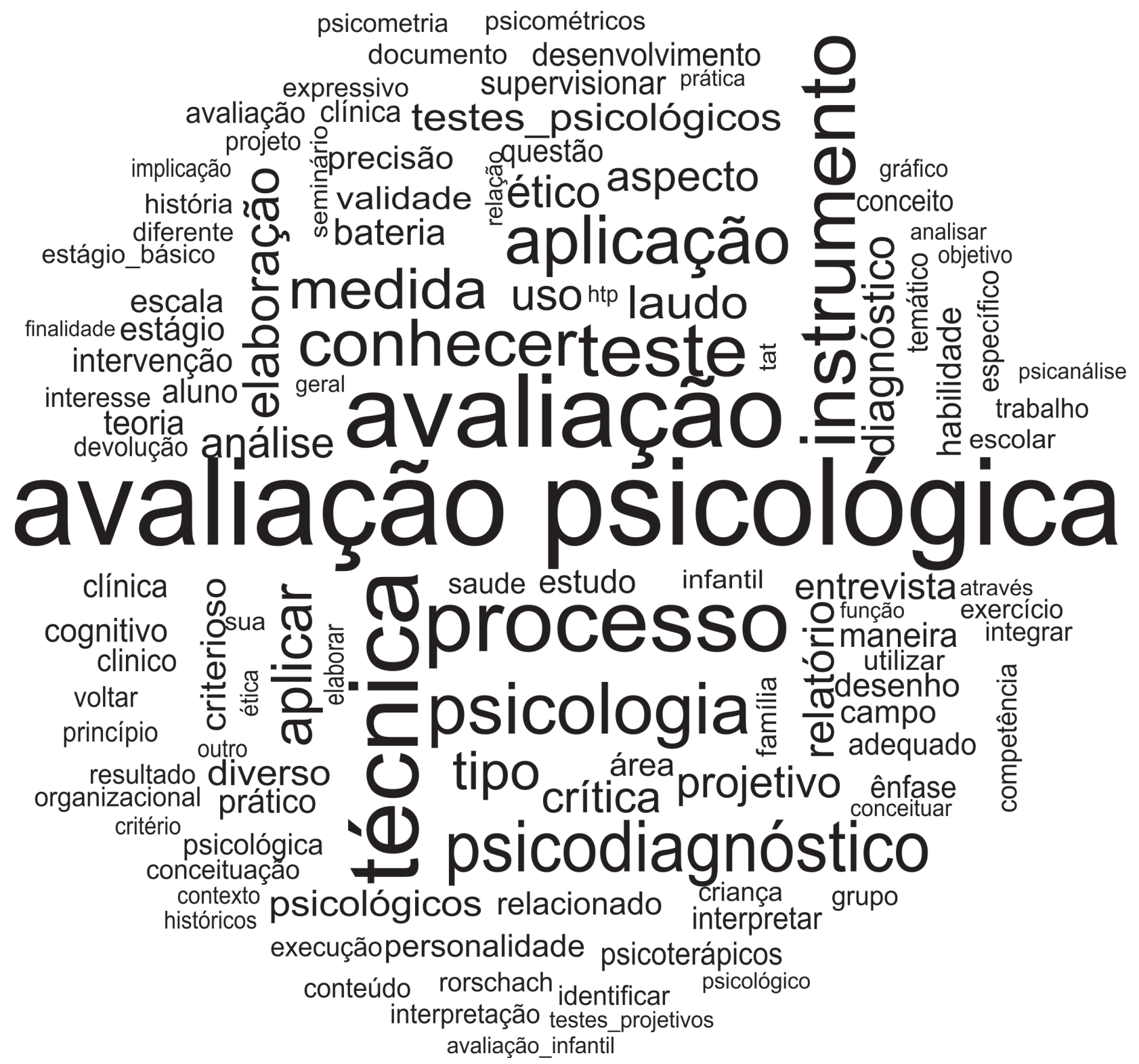

Figura 2. Nuvem de palavras do corpus 1 
demais cursos analisados ainda não é a ideal. A propósito, em uma das instituições observou-se um cenário crítico, não sendo detectada em sua matriz curricular uma disciplina específica da área de AP.

Quanto aos conteúdos ministrados nas disciplinas, observou-se a preocupação dos cursos em torno do processo psicodiagnóstico, perceptível por ser a disciplina mais mencionada. Além disso, 19,1\% de todo o conteúdo textual das ementas analisado foi relativo a elementos que perpassam o processo de AP em contexto clínico. Tais resultados são similares aos encontrados por Castro (2013), que avaliou ementas em AP de cursos no estado de São Paulo e verificou que os conteúdos mais frequentes foram aqueles relacionados ao psicodiagnóstico. Sabendo desse foco no psicodiagnóstico, é importante ter em conta o que vem sendo produzido e debatido atualmente na área. Recentemente, Krug, Trentini e Bandeira (2016) propuseram a revisão do termo psicodiagnóstico, procurando abranger formas variadas de realização desse procedimento investigativo clínico a partir de diferentes teorias psicológicas.

Ainda segundo Krug et al. (2016), a revisão da conceituação do psicodiagnóstico representa uma reflexão importante diante do que tem sido apresentado nos principais manuais da área (Arzeno, 1995; Cunha, 2000; Ocampo \& Arzeno, 2009) - sobretudo no que diz respeito à obrigatoriedade do uso de testes psicológicos - e constitui um debate relevante a ser incluído no contexto da formação em AP.

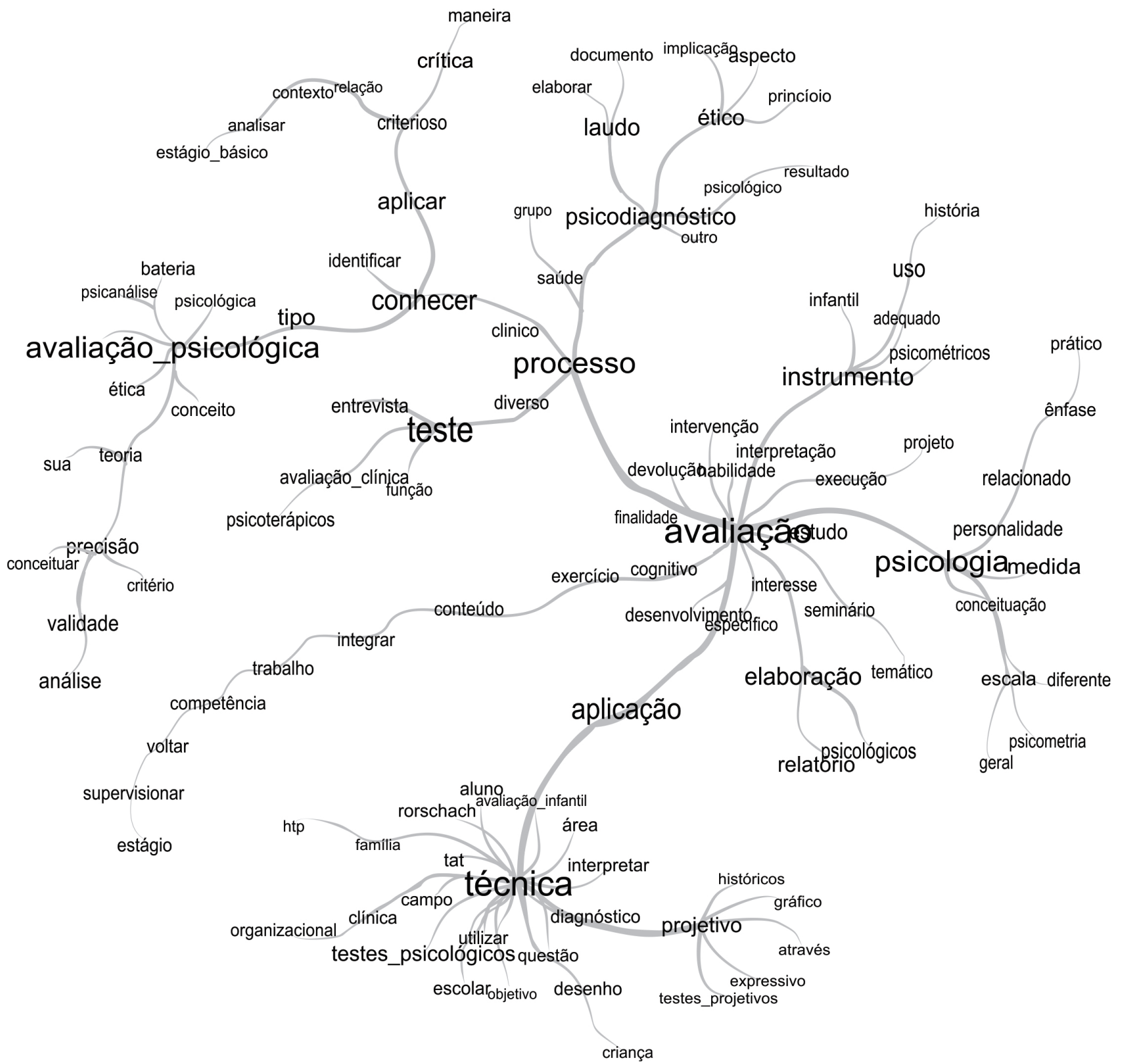

Figura 3. Árvore de coocorrências - análise de similitude do corpus 1 
Termos mais técnicos tiveram notório espaço na "nuvem de palavras", o que pode indicar que alguns cursos ainda carregam o foco tecnicista em sua formação, conforme sugerido pelo número frequente de disciplinas nomeadas como Técnicas de Exame Psicológico. Tais resultados são corroborados por Noronha, Castro, Ottati, Barros e Santana, que, em estudo com docentes, constataram que os instrumentos de AP ocupavam destaque entre os conteúdos ministrados nas disciplinas, reforçando o ensino a partir de um prisma eminentemente técnico e distante da postura crítica frente ao instrumental (Noronha \& Reppold, 2010). Portanto, tal como pontua Noronha (2006), muitos cursos de Psicologia têm ensinado a aplicar e interpretar os escores de um teste, mas poucos discutem as razões de sua aplicação.

Analisando o corpus oriundo das ementas, observa-se que a fundamentação em psicometria é um tópico secundário na formação em AP no Norte. Especificamente na "nuvem de palavras" e na análise de similitude, termos da psicometria foram quase omitidos. Analisando a classe que os agrupa, verifica-se que o conteúdo ainda está associado basicamente a noções clássicas de validade e precisão, não tendo sido mencionadas análises que focam os itens (e.g., dificuldade, discriminação, funcionamento diferencial), omitindo-se, por exemplo, a Teoria de Resposta ao Item. Esses dados sugerem que, na região Norte, enfatiza-se a Teoria Clássica dos Testes, deixando descobertos conteúdos mais recentes (e.g., equalização de testes, invariância de estruturas fatoriais), o que reforça a importância da qualidade da formação básica na área, bem como da formação continuada ofertada pelas IESs (Borsa, 2016).

É importante ressaltar, também, que pouca ênfase foi dada ao histórico da AP, tal como verificado em estudo de Noronha et al. (2013), no qual os conteúdos relacionados ao histórico constituíram um dos menos frequentes nas ementas das disciplinas de AP. Tais resultados são preocupantes em função do conhecimento de aspectos históricos, em contexto nacional e internacional, ser uma das competências básicas a serem desenvolvidas durante a formação em Psicologia (Nunes et al., 2012). Reitera-se que a formação oferecida em AP foi direcionada principalmente ao processo psicodiagnóstico, enfatizando a aplicação e a interpretação de instrumentos, e que os construtos mais abordados foram a inteligência e a personalidade. Portanto, demanda-se uma revisão cuidadosa e atualizada do currículo nessa área de estudo e atuação.

A presente pesquisa teve o objetivo de retratar a estrutura do ensino de AP na região Norte do Brasil. Contudo, as possibilidades não se esgotam aqui. Concretamente, há muito o que conhecer sobre o processo de ensino-aprendizagem nessa área. Uma das possibilidades é avaliar o domínio dos docentes dessa região acerca do campo da avaliação e de seus conteúdos específicos, verificando se estes apresentam o conhecimento necessário para transmitir os conteúdos fundamentais referentes, procurando, por exemplo, oferecer melhores oportunidades de formação continuada ou capacitação em habilidades centrais da área. Nesse contexto, reconhece-se que, provavelmente, a maior parte dos cursos de Psicologia no Brasil está longe de atingir os requisitos mínimos para o ensino apropriado em AP (Borsa, 2016). Os resultados do presente estudo parecem reforçar essa conclusão, embora muito ainda precise ser conhecido, incluindo dados de outras regiões do Brasil, principalmente Nordeste e Centro-Oeste. Essas novas informações podem acrescentar aspectos importantes para a compressão da temática no contexto nacional, complementando os achados substanciais de pesquisas já realizadas no eixo Sul-Sudeste do país.

Por fim, apesar de suas contribuições, ressalta-se que a presente pesquisa não está isenta de limitações. Um aspecto importante a destacar é que não foi analisada a totalidade das ementas dos cursos da região Norte, mas tão somente aquelas disponibilizadas nos endereços eletrônicos das instituições. Além disso, não se obteve acesso à matriz curricular de todos os cursos de Psicologia do Norte e não foi feita distinção quanto à natureza das disciplinas (obrigatórias ou optativas), tendo a atenção sido direcionada para a análise do conteúdo do ementário. Não obstante, considera-se igualmente importante que tal aspecto seja retomado e pormenorizado em estudos futuros que levem em consideração essa temática. Contudo, para além das limitações, os achados deste e de outros estudos que tiveram em conta diferentes regiões brasileiras apontam para a necessidade de criar uma estrutura curricular comum em AP, favorecendo a formação sólida e atualizada do psicólogo no país (Noronha et al., 2008; Nunes et al., 2012).

\section{Referências}

Arzeno, M. E. G. (1995) Psicodiagnóstico Clínico: novas contribuições. Porto Alegre: Artes Médicas.

Bardagi, M. P., Teixeira, M. A. P., Segabinazi, J. D., Schelini, P. W., \& Nascimento, E. (2015). Ensino da avaliação psicológica no Brasil: Levantamento com docentes de diferentes regiões. Avaliação Psicológica, 14(2), 253-260.

Borsa, J. C. (2016). Considerações sobre a formação e a prática em avaliação psicológica no Brasil. Temas em Psicologia, 24(1), 131-143. doi: 10.9788/TP2016.1-09

Camargo, B. V. (2005). Alceste: Um programa informático de análise quantitativa de dados textuais. In A. S. P. Moreira (Org.), Perspectivas teórico-metodológicas em representações sociais (pp. 511-539). João Pessoa: UFPB/Editora Universitária.

Castro, P. F. (2013). Caracterização do ensino de avaliação psicológica no estado de São Paulo. Boletim de Psicologia, 63(138), 81-102. 
Cunha, J. A. (2000) Psicodiagnóstico-V. 5a. Ed. rev. Porto Alegre: Artes Médicas.

Gouveia, V.V., Silva, D. V., Silva, M. P. V., Andrade, M. W. C. L., Silva Filho, S. B., \& Costa, D. M. F. (2002). Atitudes frente à avaliação psicológica para condutores: Perspectivas de técnicos, estudantes de psicologia e usuários. Psicologia: Ciência e Profissão, 22(2), 50-59. doi: 10.1590/S1414-98932002000200007

Krug, J. S., Trentini, C. M., \& Bandeira, D. R. (2016). Conceituação de psicodiagnóstico na atualidade. In: C. S. Hutz, D. R. Bandeira, C.M. Trentini, \& J.S. Krug. (Org.), Psicodiagnóstico (pp.16-20). Porto Alegre, RS: Artmed.

Lisboa, F. S., \& Barbosa, A. J. G. (2009). Formação em psicologia no Brasil: Um perfil dos cursos de graduação. Psicologia: Ciência e Profissão, 29(4), 718-737. doi: 10.1590/S1414-98932009000400006

Mendes, L. S., Nakano, T. C., Silva, I. B., \& Sampaio, M. H. L. (2013). Conceitos de avaliação psicológica: Conhecimento de estudantes e profissionais. Psicologia: Ciência e Profissão, 33(2), 428-445. doi: 10.1590/S1414-98932013000200013

Noronha, A. P. P. (2006). Formação em avaliação psicológica: Uma análise das disciplinas. Interação em Psicologia, 10, 245-252. doi: 10.5380/ psi.v10i2.7681

Noronha, A. P. P., \& Reppold, C. T. (2010). Considerações sobre a avaliação psicológica no Brasil. Psicologia: Ciência e Profissão, 30(spe), 192-201. doi: 10.1590/S1414-98932010000500009

Noronha, A. P. P., Baldo, C. R., Barbin, P. F., \& Freitas, J. V. (2003). Conhecimento em avaliação psicológica: Um estudo com alunos de Psicologia. Psicologia: Teoria e Prática, 5(2), 37-46.

Noronha, A. P. P., Barros, M. V. C., Nunes, M. F. O., \& Santos, A. A. A. (2014). Avaliação psicológica: Importância e domínio de atividades segundo docentes. Estudos e Pesquisas em Psicologia, 14(2), 524-538.

Noronha, A.P. P., Batista, M. A., Carvalho, L., Cobêro, C., Cunha, N. B., Dell'Aglia, B. A. V., Filizatti, R., Zenorini, R. P. C., \& Santos, M. M. (2008). Ensino de avaliação psicológica em Instituições de Ensino Superior brasileiras. Universitas Ciências da Saúde, 3(1), 1-14. doi: 10.5102/UCS.V3I1.543

Noronha, A. P. P., Carvalho, L. F., Miguel, F. K., Souza, M. S., \& Santos, M. A. (2010). Sobre o ensino da avaliação psicológica. Avaliação Psicológica, 9(1), 139-146.

Noronha, A. P. P., Castro, N. R., Ottati, F., Barros, M. V. C., \& Santana, P. R. (2013). Conteúdos e metodologias de ensino de avaliação psicológica: Um estudo com professores. Paidéia, 23(54), 129-139.

Noronha, A. P. P., Nunes, M. F. O., \& Ambiel, R. M. (2007). Importância e domínios de avaliação psicológica. Um estudo com alunos de psicologia. Paidéia, 17(37), 231-244.

Nunes, M. F. O., Muniz, M., Reppold, C. T., Faiad, C., Bueno, J. M. H., \& Noronha, A. P. P. (2012). Diretrizes para o ensino da avaliação psicológica. Avaliação Psicológica, 11(2), 309-316.

Ocampo, M. L. S., \& Arzeno, M. E. G. (2009). A entrevista inicial. Em M. L. S. Ocampo, M. E. G. Arzeno, \& E. G. Piccolo (Eds.), O processo psicodiagnóstico e as técnicas projetivas. São Paulo: Martins Fontes.

Padilha, S., Noronha, A. P. P., \& Fagan, C. S. (2007). Instrumentos de avaliação psicológica: Uso e parecer de psicólogos. Avaliação Psicológica, 6(1), 69-76.

Ratinaud, P. (2009). IRAMUTEQ: Interface de R pour les Analyses Multidimensionnelles de Textes et de Questionnaires [Computer software]. Recuperado de http://www.iramuteq.org

Ratinaud, P., \& Marchand, P. (2012). Application de la méthode ALCESTE à de "gros" corpus et stabilité des "mondes lexicaux": analyse du "CableGate" avec IraMuTeQ. In: Actes des 11eme Journées internationales d’Analyse statistique des Données Textuelles (pp. 835-844). Liège, Belgique. Recuperado de http://lexicometrica.univ-paris3.fr/jadt/jadt2012/Communications/Ratinaud,\%20Pierre\%20et20al.\%20-\%20 Application\%20de\%20la\%20methode\%20Alceste.pdf

Paula, A. V., Pereira, A. S., \& Nascimento, E. (2007). Opinião de alunos de psicologia sobre o ensino em avaliação psicológica. Psico-USF, 12(1), 33-43.

Primi, R. (2010). Avaliação psicológica no Brasil: Fundamentos, situação atual, e direções para o futuro. Psicologia: Teoria e Pesquisa, 26(spe), 25-35. doi: 10.1590/S0102-37722010000500003

Primi, R., Hutz, C. S., \& Silva, M. C. R. (2011). A prova do ENADE de psicologia 2006: Concepção, construção e análise psicométrica da prova. Avaliação Psicológica, 10(3), 271-294.

R Development Core Team (2012). R: A language and environment for statistical computing. R Foundation for Statistical Computing, Vienna, Austria. Recuperado de http://www.R-project.org/.

Reppold, C. T., \& Serafini, A. J. (2010). Novas tendências no ensino da avaliação psicológica. Avaliação Psicológica, 9(2), 323-329.

Santos, A. M., Anache, A. A., \& Santana, R. C. (2015). Panorama da produção científica brasileira em avaliação psicológica. Psico-USF, 20 (3), 547-559.

Souza, M. P. R., Bastos, A. V., \& Barbosa, D. R. (2011). Formação básica e profissional do psicólogo: Análise do desempenho dos estudantes no ENADE-2016. Avaliação Psicológica, 10(3), 295-312.

\section{Sobre os autores}

Leogildo Alves Freires é psicólogo, possui mestrado e doutorado em Psicologia Social pela Universidade Federal da Paraíba. Atual mente é professor adjunto do curso de Psicologia da Universidade Federal de Roraima.

José Humberto da Silva Filho é psicólogo, possui doutorado em Psicologia pela Universidade de São Paulo. Atualmente é professor adjunto do curso de Psicologia da Universidade Federal do Amazonas.

Renan Pereira Monteiro é psicólogo, possui mestrado e doutorado em Psicologia Social pela Universidade Federal da Paraíba. Atualmente é professor substituto do curso de Psicologia da Universidade Federal do Piaú.

Gleidson Diego Lopes Loureto é psicólogo, possui graduação em Psicologia pela Universidade Federal de Roraima. Atualmente é mestrando em Psicologia Social pela Universidade Federal da Paraíba.

Valdiney Veloso Gouveia é psicólogo, possui doutorado em Psicologia Social pela Universidad Complutense de Madrid. Atualmente é professor titular do Programa de Pós-Graduação em Psicologia Social da Universidade Federal da Paraíba. 